\title{
Improved osteosarcoma survival with addition of mifamurtide to conventional chemotherapy - a prospective single institution analysis.
}

Peter Múdry ( $\square$ mudry.peter@fnbrno.cz )

Fakultni nemocnice Brno Detska nemocnice

Ondřej Rohleder

Fakultni nemocnice Brno Detska nemocnice

Michal Kýr

Fakultni nemocnice Brno Detska nemocnice

Jaroslav Štěrba

Fakultni nemocnice Brno Detska nemocnice

Research article

Keywords: osteosarcoma, mifamurtide, event and progression free survival, single institution analysis, conventional chemotherapy

Posted Date: September 25th, 2019

DOI: https://doi.org/10.21203/rs.2.14968/v1

License: (9) This work is licensed under a Creative Commons Attribution 4.0 International License. Read Full License 


\section{Abstract}

Background Conventional osteosarcoma is rare disease. Current treatment approaches have been set decades ago and include combination of three drug chemotherapy schedule and surgery. Three and five years event free survival in localized disease is roughly $59-54 \%$, respectively. An immunotherapy agent mifamurtide was introduced to clinical practice after registration study which reported survival benefit, but some methodological controversies have been unsufficient for Food and Drug Administration market authorization

Methods We report here prospective single center analysis of mifamurtide addition to conventional therapy in 16 patients during 5.5 year enrollment period.

Results Adverse event profile was as expected with no dose limiting toxicities. All patients were given mifamurtide according to planned treatment schedule. There were no local relapses observed, one patient died in first complete remission due to doxorubicine cardiotoxicity, one patient had pulmonary metastatic relapse. Observed three and five years event free survival was 82,6 (Cl 70-100\%) and 82,6\% (Cl 70-100\%), progression free survival was $92,3 \%(\mathrm{Cl} 78,9-100 \%)$ and $92,3 \%(\mathrm{Cl} 78,9-100 \%)$, overall survival was 93,3 (Cl 81,5-100) and 74,7 (Cl 47,2-100), respectively.

Conclusions Survival benefit of mifamurtide is reported herein. Addition of mifamurtide should be considered as best treatment option for patients with localized osteosarcoma.

\section{Background}

Osteosarcoma is the most common malignant mesenchymal tumor of the bone in pediatric population. That can arise from any bone, but most often localization of tumor's origin is in metafyseal area of long bones. Osteosarcoma is composed of malignant osteoblasts, which produce immature bone tissue and osteoid. In histological point of view osteosarcoma can be subdivided into high grade forms such as conventional, telangiectatic or chondroblastic and into more indolent forms like parosteal or periosteal osteosarcoma.

Incidence of that disease is very rare of $4.0(3.5-4.6)$ for the range $0-14$ years and $5.0(4.6-5.6)$ for the range $0-19$ years per year per million persons and it varies with age (1). There are well known two incidence peaks. First during skeletal growth spurt in age of 15-19 years and second one is during elderly age. (1)Known environmental and genetic risk factors are previous irradiation of the bone, Pagets disease or heritable syndromes such as Li-Fraumeni or germline mutation in RB1 tumor suppressor gene.

Clinical presentation of osteosarcoma is predominantly pain in affected area which initially appears after physical activities and afterwards typically during sleep in "on/off" mode. In general, further symptoms are swelling, palpable mass or loss of function of the limb. Up to $20 \%$ of patients are diagnosed with apparent metastatic disease (lungs, bones), but majority of patients are diagnosed with localized disease. Despite that, they have already microscopic metastases in time of the diagnosis. (2) 
Total resection of clinically detectable tumors is mainstay of long term survival, even if treatment approach for this disease needs to be multimodal. Though methods of surgical local control were improved and shifted more to limb salvage strategy than previously used amputations, relaps-free survival rates of localized extremity tumors are still approximately $15-17 \%$ in case of surgical treatment only. Combination of surgery with intensive neoadjuvant and adjuvant multiagent chemotherapy controls microscopic metastatic disease and improve free-survival rates to about $70 \%$. Nevertheless, in cases of metastatic disease, local progression or nonresectable primary tumors the success rate is still very poor with less than $20 \%$ survival five years since diagnosis. (3) Since 70 's of previous century till nowadays only four chemotherapeutic agents have been routinely used in systemic therapy. Backbone of conventional chemotherapy treatment is built by doxorubicin, cisplatine and methotrexate with leucovorin rescue. An alkylation agent ifosfamide is also effective in treatment of osteosarcoma but addition of ifosfamide to other agents is too toxic and did not improve treatment results. Up to date no any new chemotherapy agents with proven efficacy have been added to standard chemotherapy regimen and overall survival rates are stagnating. Thus, treatment strategy for locoregional disease is still based on former schedules. Other additional and experimental treatments in metastatic or progressive disease consist also of targeting the bone microenvironment (bisfosfonates), tyrosin kinase receptors (e.g. sorafenib, pazopanib), intracellular signaling molecules (dasatinib) and of unspecific immune therapy (interferon).

Mifamurtide (Mepact) is synthetic lipophilic analog of muramyl dipeptid, peptidoglycan contained in bacterial wall, which can activate innate immune system. Its tumoricidal effect on microscopic metastases is probably caused by stimulation of monocytes and macrophages associated with increasing level of proinflammatory cytokines. The rationale behind the use of mifamurtide in osteosarcoma treatment is to mimic a kind of infection that can help to eradicate residual micrometastases that are not eliminated by systemic chemotherapy.(2) View of clinical community on regular administration of mifamurtide with standard adjuvant chemotherapy is still not endorsed despite registration study which showed benefit of mifamurtide addition to adjuvant chemotherapy and significantly improved 6-year overall survival from $70 \%$ to $78 \%$, event free survival was $61 \%$.(4)

\section{Methods}

Since middle 2012 till the end of 2018 we diagnosed 16 patients with localized osteosarcoma. Eight out of 16 diagnosed patients (50\%) were in adolescent age, male:female ratio was 11:5 and most common sites were proximal humerus $(31 \%)$ and proximal tibia $(25 \%)$. Six of them $(37,5 \%)$ had unspecific nodules in lungs at diagnosis, three of them with nodules size in range 1-5 mm, other three with size of 6-9 mm. Histologically, 12 out of $16(75 \%)$ tumors were conventional osteoblastic type, rest of them belonged to other histological subgroups of osteosarcoma, one to each type-teleangiectatic, chondroblastic, smallcell and sclerosing (Table1). Age distribution of patients is shown in Figure 1.

All of diagnosed patients were enrolled to regimen with mifamurtide including one with relapsed locoregional disease. Mifamurtide was administrated intravenously as 1 hour infusion in dose of 
$2 \mathrm{mg} / \mathrm{m} 2$ (maximum $4 \mathrm{mg}$ single dose). Total of 48 doses were given to each patient, 24 doses twice a week and 24 doses once a week during 36 weeks. As premedication we routinely used paracetamol in dose of $500-1000 \mathrm{mg}$.

Sample characteristic was given in summary tables. Outcome of the patients was analyzed using survival analysis. Results were shown as Kaplan-Meier curves and summarized as estimates for 3- and 5year survivals. White blood cell counts recovery was shown as cumulative incidence. Analyses were done using R software 3.5.1 (5).

\section{Results}

After neoadjuvant treatment, 9 of $16(56 \%)$ achieved good response defined as necrosis $\geq 90 \%$ and 4 of $16(25 \%)$ had poor response on treatment. Three out of 16 patients $(18 \%)$ underwent surgical resection before administration of chemotherapy thus their histologic response was not evaluable. Only one out of four poor responders later died of progressive metastatic disease.

We observed some of known side effects during administrations of mifamurtide. These were fever and chills in majority of patients (Table 2). One of the patients had myalgia/lumbalgia after first dose of mifamurtide and another one had several times nausea and vomiting. Relevance of adverse events was predominantly grade I or grade II (according to CTCAE criteria v3.0) and all of them resolved successfully after symptomatic medication. In general, most of the patients tolerated administration of mifamurtide really well. All patients were given full doses of mifamurtide as scheduled. Recovery of leukocytes afterward last chemotherapy was 2,6 weeks.

With a median follow-up period of 41,8 months, 14 out of 16 patients $(87,5 \%)$ are alive and in complete remission. One patient died of heart failure during secondary myelodysplastic syndrome, one patient had metastatic progression to lung and died eventually. None of patients had locoregional progression. The observed event free survival at 3 years was $86,2 \%$ (Cl 70-100\%), overall survival at 3 years was $93,3 \%$ (Cl $81,5-100 \%)$, progression free survival at 3 years was $92,3 \%(\mathrm{Cl} 78,9-100 \%)$. These and 5 -year survival estimates are shown in Table 3 and Figure 3.

\section{Discussion}

To our knowledge we report herein the best event and progression free survival results of treatment of locoregional osteosarcoma published. Our study has some limitations. First of all, the sample size is small. Second, sample size needs one and half more year for robust 5-year survival estimates to conclude that 3-year estimate survivals will be maintained. Locoregional control with no any progressive disease describes well experienced team of orthopedic surgeons. Pediatric oncologists, orthopedic surgeons, pathologist and radiologists in our center have set a multidisciplinary board with regular meetings. All specialists discuss the disease management issues for each patient - about timing and type of surgery and coordination with chemotherapy or adjuvant radiotherapy-to minimalize treatment protocol 
deviations. Both chemotherapy and mifamurtide schedules were kept. Long lasting chemotherapy has adverse effect to bone recovery. Leukocyte recovery with median 2,6 weeks is expected and not compromised by mifamurtide.

Recent analysis of Euramos -1 study which was conducted worldwide and included 2260 patients demonstrated the 3-year and 5-year event free survival 59 and 54\%, and overall survival 79 and $71 \%$ respectively. This study confirmed previously reported results of other cooperative groups or institutional series. (6)

For evaluation of mifamurtide efficacy is better to use 3-year and 5-year metastatic free survival. We report herein progression free survival 92,3\% (Cl 78,9-100\%) and 92,3\% (Cl 78,9-100\%), respectively, which is equivalent to metastatic free progression.

Also another small size study confirms better treatment result with mifamurtide as 3-year overall and event free survival 87,5 and $75,6 \%$, respectively. (7)

Compared to our small sample size, the large French study reports in 126 patients survival benefit of mifamurtide as $18 \%$ improvement in 3-year event free survival ( 52 vs.70\%, HR 0,55), even worse than in our analysis and comparable to Euramos results without mifamurtide.(8)

For patients with metastatic disease, the published results show trend to longer overall and event free survival after addition of mifamurtide, but phase III sample size was small and improvement did not achieved conventional statistical significance. Results of an expanded access trial suggest a decreased risk of subsequent recurrence and death with the inclusion of mifamurtide in the treatment strategy for metastatic high-risk patients also. (9)

\section{Conclusions}

We conclude that combination of chemotherapy, surgery and mifamurtide in adjuvant setting is the best treatment option for young patients with osteosarcoma. Experienced multidisciplinary advisory task board is essential in each center which cares of osteosarcoma patients. The expert team should give every effort at all times to avoid protocol deviations and keep treatment schedules as recommended.

\section{Abbreviations}

CTCAE common toxicity criteria for adverse events

Cl confidence interval

EFS event free survival

HR hazard ratio 
OS overall survival

PFS progression free survival

\section{Declarations}

\section{Ethics Approval and Consent to Participate}

The treatment described in this study consisted of "on-label" drugs and prospective data collection has been approved by institutional ethics committee of University Hospital Brno. All research described herein was conducted according to the Declaration of Helsinki. The written parental or participant (in patients over age of 18 years, according to national regulations) consent with therapy was obtained.

Consent for Publication

Not Applicable

Availability of data and material

The datasets used and/or analysed during the current study are available from the corresponding author on reasonable request.

Authors' contributions

All authors have read and approved the manuscript.

PM contributed to study conception and design, data acquisition and interpretation and wrote the manuscript

OR contributed to data acquisition and wrote the manusript

MK contributed to data analysis and interpretation

JS contributed to study conception and design

Competing interests

The authors disclose no potential conflicts of interest.

Funding

Supported by the European Regional Development Fund - Project ENOCH (No.

CZ.02.1.01/0.0/0.0/16_019/0000868) and No 16-34083A from the Ministry of Healthcare of the Czech Republic. The funding body supported data analysis, manusript preparation and publication fees. 
Acnowledgements: Full coverage of treatment expenses by insurance companies of each respective patient included in this manusript is appreciated.

\section{References}

1. Ottaviani G, Jaffe N. The epidemiology of osteosarcoma. Cancer Treat Res. 2009;152:3-13.

2. Kager L, Pötschger U, Bielack S: Review of mifamurtide in the treatment of patients with osteosarcoma, Therapeutics and Clinical Risk Management. 2010; 6:279-86.

3. Kansara M, Teng MW, Smyth MJ, Thomas DM: Transitional biology of Osteosarcoma, Nature reviews, vol.14, 2014.

4. Chou AJ, Kleinerman ES, Krailo MD, et al. Addition of muramyl tripeptide to chemotherapy for patients with newly diagnosed metastatic osteosarcoma: a report from the Children's Oncology Group. Cancer. 2009;115:5339-48.

5. R Development Core Team (2008). R: A language and environment for statistical computing. $R$ Foundation for Statistical Computing, Vienna, Austria. ISBN 3-900051-07-0, URL http://www.Rproject.org.

6. Smeland S, Bielack SS, Whelan J., et al. Survival and prognosis with osteosarcoma: outcomes in more than 2000 patients in the EURAMOS-1 (European and American Osteosarcoma Study) cohort. European Journal of Cancer.2019; 109: 36-50.

7. Tacyildiz N, Incesoy Ozdemir S, Unal E, et al. The Efficiency and Toxicity of Mifamurtide in Childhood Osteosarcoma. Journal of pediatric hematology/oncology. 2018; 40. e373-e376.

8. Brard C, Piperno-Neumann S, Delaye J. Sarcome-13/0S2016 trial protocol: a multicentre, randomised, open-label, phase II trial of mifamurtide combined with postoperative chemotherapy for patients with newly diagnosed high-risk osteosarcoma. BMJ Open, 2019;9:e025877.

9. Meyers PA, Chou AJ. Muramyl tripeptide-phosphatidyl ethanolamine encapsulated in liposomes (LMTP-PE) in the treatment of osteosarcoma. Adv Exp Med Biol. 2014;804:307-21.

\section{Tables}

Table 1. Patients characteristics 


\begin{tabular}{|c|c|c|}
\hline & Number & $\%$ \\
\hline Patients & 16 & 100 \\
\hline Sex Male & 11 & 69 \\
\hline Female & 5 & 31 \\
\hline Age at diagnosis & 13,7 years & - \\
\hline Histology & & \\
\hline Conventional (osteoblastic) & 12 & 75 \\
\hline Other ${ }^{1}$ & 4 & 25 \\
\hline Patients with unspecific lung nodules & & \\
\hline Size of 1-4 mm & 3 & 19 \\
\hline Size of 5-9 mm & 3 & 19 \\
\hline Tumor localisation & & \\
\hline Humerus & 5 & 31 \\
\hline Tibia & 5 & 31 \\
\hline Femur & 2 & 13 \\
\hline Fibula & 2 & 13 \\
\hline Axial (skull, scapula) & 2 & 13 \\
\hline Histologic response & & \\
\hline Good & 9 & 56 \\
\hline Poor & 7 & 44 \\
\hline
\end{tabular}

1. each one - chondroblastic, teleangiectatic, sclerosing, small-cell

Table 2. Mifamurtide adverse events

\begin{tabular}{|l|c|c|}
\hline Adverse event & Number of patients & $\%$ \\
\hline None & 3 & 19 \\
\hline Fever only & 1 & 6 \\
\hline Fever and chills & 8 & 51 \\
\hline Headache only & 1 & 6 \\
\hline Fever, chills and headache & 1 & 6 \\
\hline Fever, chills and abdominal pain & 1 & 6 \\
\hline Fever, chills and myalgia & 1 & 6 \\
\hline
\end{tabular}

Table 3. Survival analysis

\begin{tabular}{|l|c|c|}
\hline & 3-year survival (CI) \% & 5-year survival (CI) \% \\
\hline Event free survival & $86,2(70-100) \%$ & $86,2(70-100) \%$ \\
\hline Overall survival & $93,3(81,5-100) \%$ & $74,7(47,2-100) \%$ \\
\hline Progression free survival & $92,3(78,9-100) \%$ & $92,3(78,9-100) \%$ \\
\hline
\end{tabular}

\section{Figures}




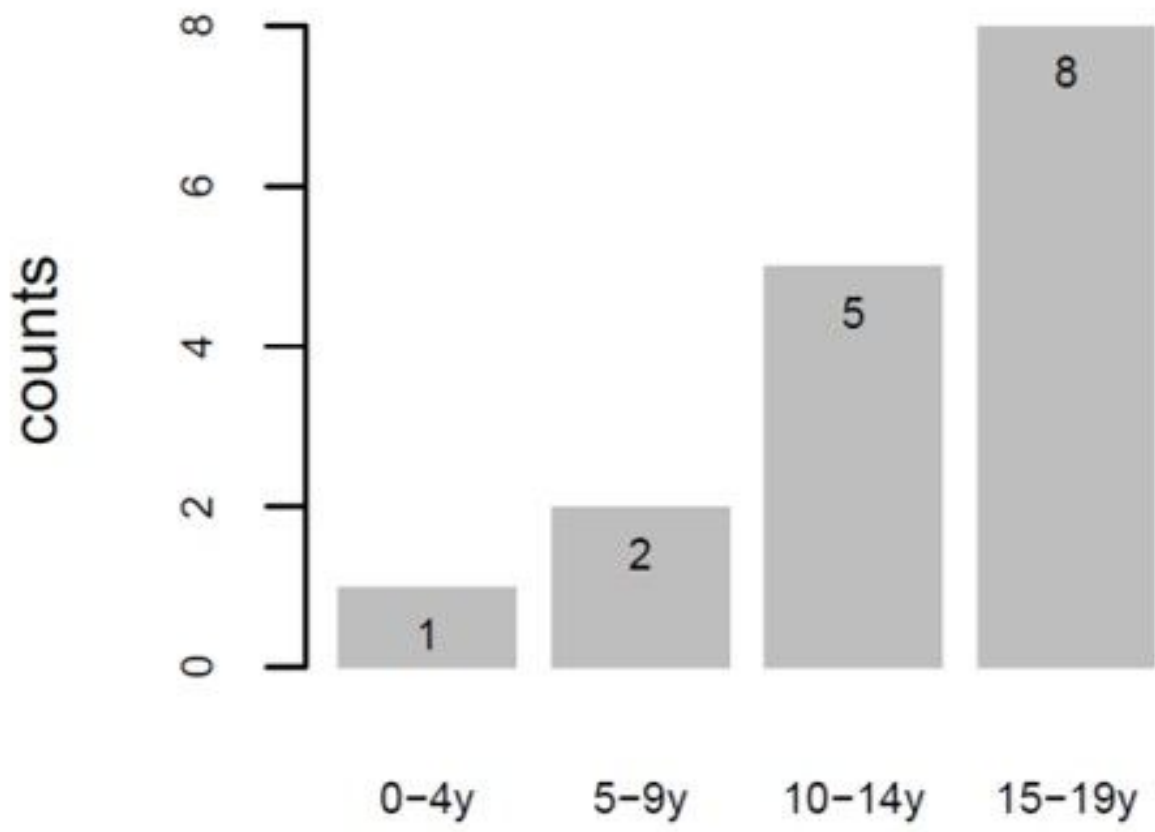

Figure 1

Patients characteristics 


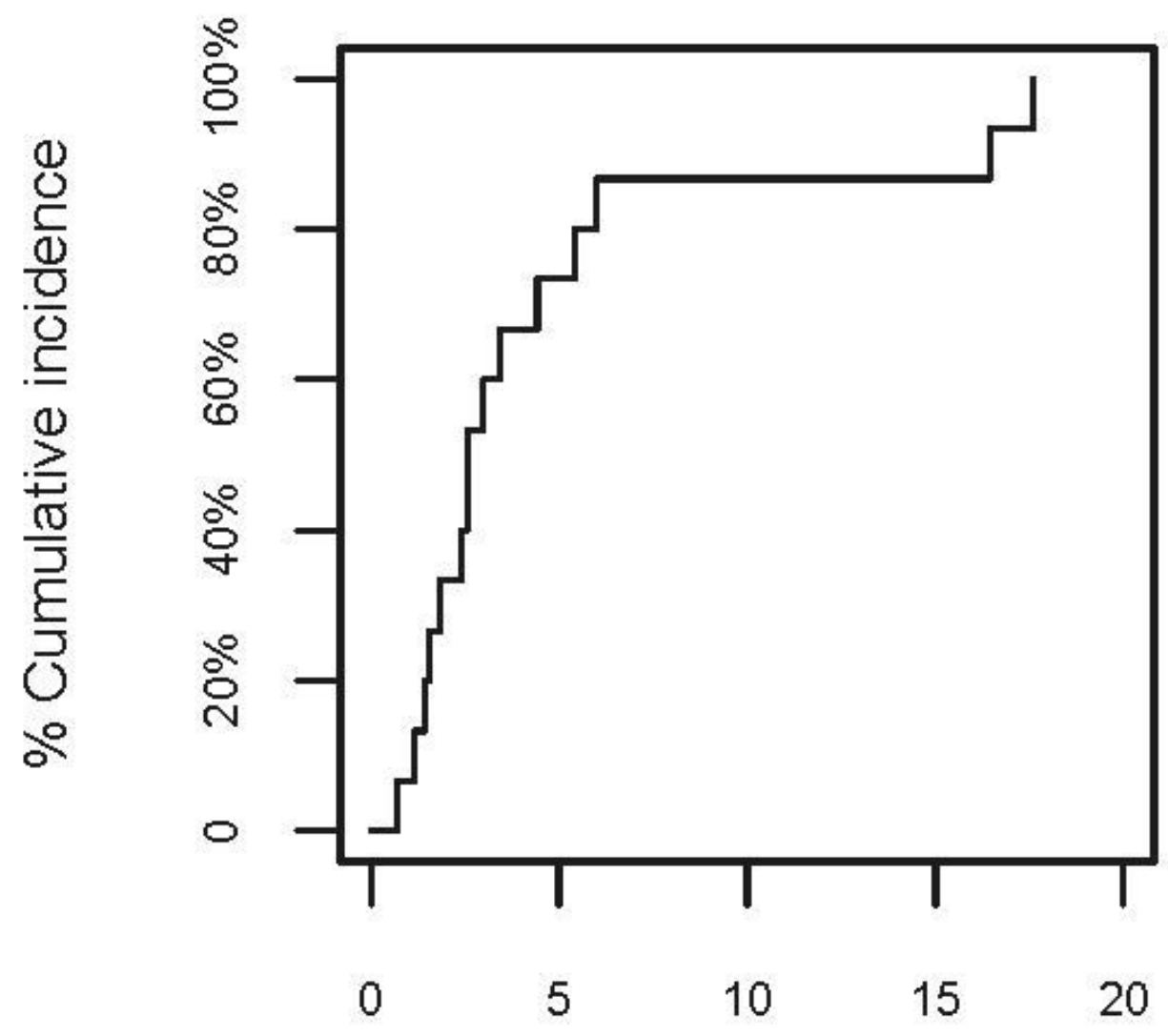

weeks

$\begin{array}{rrrr} & \text { N } & \text { 4-week (Cl) \% } & \text { 8-week (Cl) \% } \\ \text { Leu recovery } & 15 & 66.7(31.8-83.7) \% & 86.7(51.6-96.3) \%\end{array}$

Figure 2

Mifamurtide adverse events 


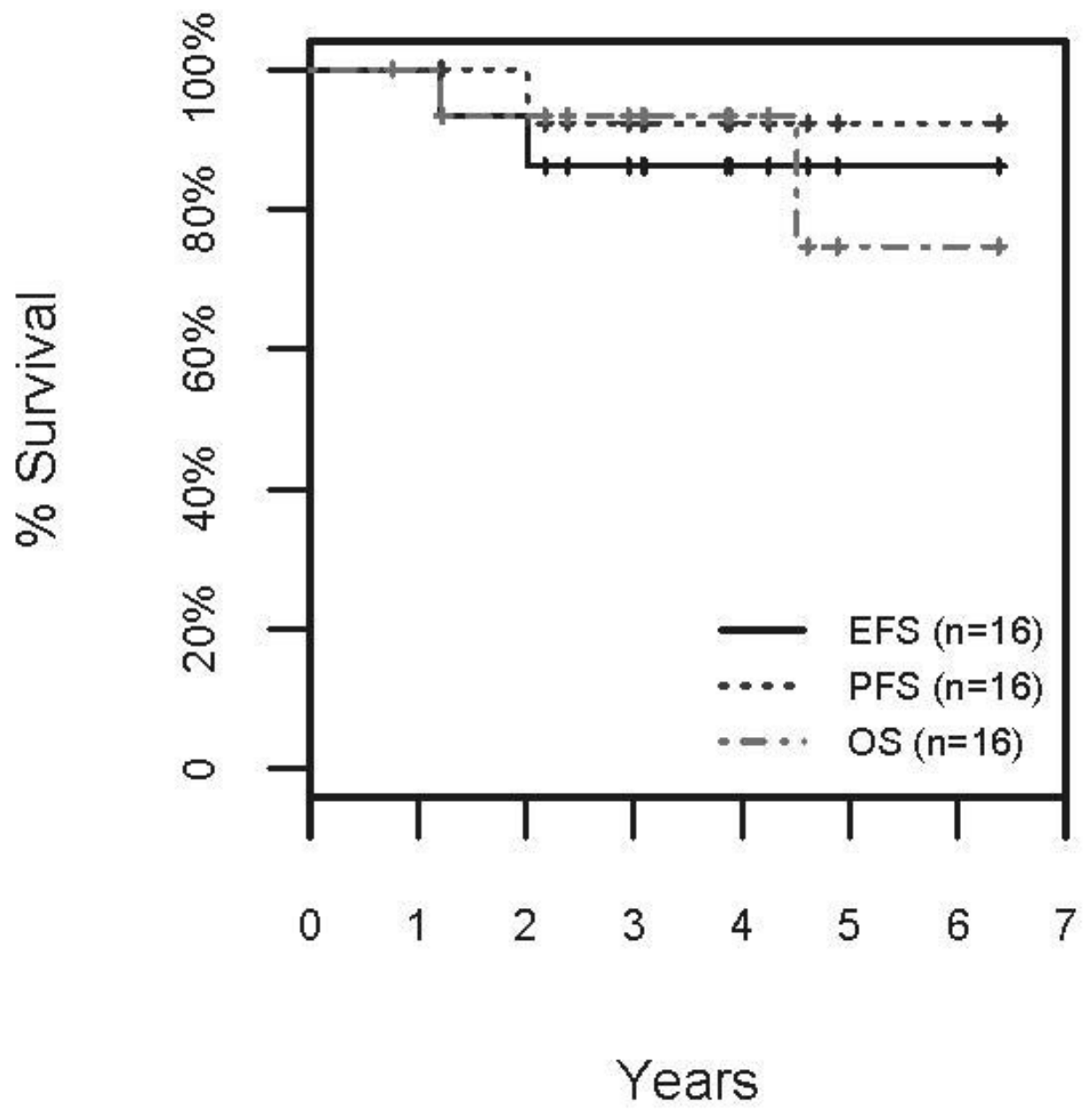

Figure 3

Survival analysis 